OPEN ACCESS

Edited by:

Joachim Hermann Schiemann,

Julius Kühn-Institut, Germany

Reviewed by:

Tao Sun,

Stanford University, United States

Lijun Chai,

Huazhong Agricultural University,

China

*Correspondence:

Nian Wang

nianwang@ufl.edu

Specialty section:

This article was submitted to

Plant Biotechnology,

a section of the journal

Frontiers in Plant Science

Received: 03 October 2017 Accepted: 01 December 2017 Published: 12 December 2017

Citation:

Jia H, Xu J, Orbović V, Zhang Y and Wang N (2017) Editing Citrus Genome via SaCas9/sgRNA System.

Front. Plant Sci. 8:2135.

doi: 10.3389/fpls.2017.02135

\section{Editing Citrus Genome via SaCas9/sgRNA System}

\author{
Hongge Jia ${ }^{1}$, Jin $\mathrm{Xu}^{1}$, Vladimir Orbović ${ }^{1,2}$, Yunzeng Zhang ${ }^{1}$ and Nian Wang ${ }^{1 *}$ \\ ${ }^{1}$ Citrus Research and Education Center, Department of Microbiology and Cell Science, Institute of Food and Agricultural \\ Sciences, University of Florida, Lake Alfred, FL, United States, ${ }^{2}$ Citrus Research and Education Center, Institute of Food and \\ Agricultural Sciences, University of Florida, Lake Alfred, FL, United States
}

SaCas9/sgRNA, derived from Staphylococcus aureus, is an alternative system for genome editing to Streptococcus pyogenes SpCas9/sgRNA. The smaller SaCas9 recognizes a different protospacer adjacent motif (PAM) sequence from SpCas9. SaCas9/sgRNA has been employed to edit the genomes of Arabidopsis, tobacco and rice. In this study, we aimed to test its potential in genome editing of citrus. Transient expression of SaCas9/sgRNA in Duncan grapefruit via Xcc-facilitated agroinfiltration showed it can successfully modify CsPDS and Cs2g12470. Subsequently, binary vector GFP-p1380N-SaCas9/35S-sgRNA1:AtU6-sgRNA2 was developed to edit two target sites of Cs7g03360 in transgenic Carrizo citrange. Twelve GFP-positive Carrizo transformants were successfully established, designated as \#Cz1 to \#Cz12. Based on targeted next generation sequencing results, the mutation rates for the two targets ranged from 15.55 to $39.13 \%$ for sgRNA1 and 49.01 to $79.67 \%$ for sgRNA2. Therefore, SaCas9/sgRNA can be used as an alternative tool to SpCas9/sgRNA for citrus genome editing.

Keywords: SaCas9, sgRNA, Citrus, genome editing, off-target

\section{INTRODUCTION}

Clustered Regularly Interspaced Short Palindromic Repeats (CRISPR)/CRISPR-associated sequences (Cas) systems provide adaptive immunity against viruses and other invading genetic materials (Garneau et al., 2010; Horvath and Barrangou, 2010). The CRISPR/Cas systems are diverse in prokaryotes and divided into Class 1 (Makarova et al., 2017a), where the effector complex is multimeric, and Class 2, which contains monomeric complex (Makarova et al., 2017b). Three types of CRISPR/Cas (types I, III, and IV) belong to Class 1, whereas types II, V, and VI are in Class 2. Among them, Cas9 in the Type II CRISPR/Cas system is a RNA-guided endonuclease that induces a double-strand break (DSB) into target genes. The specificity of Cas9 is guided by a transactivating small RNA (tracrRNA) and CRISPR RNA (crRNA). Introducing DSBs at targeted sites by Cas 9 via tracrRNA/crRNA recognition, which subsequently results in mutations through DNA repair, has been used to make genome modification. In 2012, the type II CRISPR/Cas system from Streptococcus pyogenes SF370 was adapted from a four-component to a more manageable twocomponent SpCas9/single guide RNA (sgRNA) system (Jinek et al., 2012). To date, SpCas9/sgRNA technology has been successfully exploited to modify the genomes of animal, plant, fungus, and microbe (Cho et al., 2013; Li et al., 2014; Liu et al., 2015; Altenbuchner, 2016). 
CRISPR/Cas-mediated genome editing provides a unique opportunity to accelerate and simplify the improvement of many crops which are difficult to achieve using traditional approaches. Among them, citrus is an important fruit crop grown worldwide. Traditional breeding to improve citrus traits is time-consuming and difficult due to tree size, polyembryony, pollen-ovule sterility, sexual and graft incompatibilities, and extended juvenility (Davey et al., 2005). In addition, citrus production has been devastated by Huanglongbing (HLB, or greening) in many places of the world, such as Florida (Gottwald, 2010; Wang et al., 2017). New biotechnology methods like CRISPR/Cas system, is urgently required for cultivating disease-resistant citrus cultivars. It should be noted that one of the significant advantages of CRISPR/Cas system over other citrus breeding via citrus transformation method is the precise targeting of user-singled-out genome sequences related to agronomic traits. For example, SpCas9/sgRNA system has been used successfully to produce cankerresistant citrus through disrupting canker susceptibility gene CsLOB1 or its promoter region (Jia et al., 2017; Peng et al., 2017).

Besides SpCas9/sgRNA, several other type II CRISPR/Cas systems have been exploited to target different PAM sequences other than $5^{\prime} \mathrm{NGG}$ motif, which is commonly used as SpCas9/sgRNA PAM. Among them, Staphylococcus aureusderived SaCas9/sgRNA, whose preferred PAM sequence is $5^{\prime} \mathrm{NNGRRT}$, is regarded as a promising alternative to SpCas9/sgRNA (Ran et al., 2015), since SaCas9 is similar to SpCas9 as for crystal structure and genome-editing efficiency (Nishimasu et al., 2015). Notably, SaCas9 (1053 amino acids) is smaller than SpCas9 (1368 amino acids). SaCas9 requires a natural guide sequence of 21-23 nucleotides, whereas SpCas9 natural guide sequence is 20-nucleotide. The first study of SaCas9/sgRNA confirmed that SaCas9/sgRNA could be employed to edit mouse genome in vivo in Ran et al. (2015). Subsequently, SaCas9/sgRNA system has been reported to modify plant genome, including Arabidopsis, tobacco and rice (Steinert et al., 2015; Kaya et al., 2016). In the present study, we tested SaCas9/sgRNA application in citrus genome editing. Application of SaCas9/sgRNA in citrus genome editing has potential to broaden target selection and take advantage of the smaller size of SaCas9.

\section{MATERIALS AND METHODS}

\section{Plasmid Construction}

p1380N-Cas9/sgRNA:cslob1 was digested with HindIII, and the HindIII-CsVMV-GFP-35T-HindIII fragment was sub-cloned into HindIII-digested p1380N-Cas9 to obtain GFP-p1380N-Cas9. The GFP-p1380N-Cas9/sgRNA:cslob1 and p1380N-Cas9 was described previously (Jia et al., 2017). Using primers SaCas9P1-XbaI (5'-AGGTTCTAGAGGATCCACCGGTGCCACCATG GCCCCAAAGAAGAAGCGGAAG-3') and SaCas9-P2-EcoRI (5'-AGGTGAATTCTTACTTTCCCATCAACCTGGGTCCAA G-3'), the 3333 bp SaCas9 was PCR-amplified from pX602-AAV-TBG::NLS-SaCas9-NLS-HA-OLLAS-bGHpA;U6::
BsaI-sgRNA (Addgene plasmid \#61593). After XbaI-EcoRI digestion, SaCas9 was inserted into XbaI-EcoRI-treated GFP-p1380N-Cas9 to form GFP-p1380N-SaCas9.

The sgRNA scaffold portion was amplified from pX602-AAVTBG::NLS-SaCas9-NLS-HA-OLLAS-bGHpA;U6::BsaI-sgRNA, using a pair of primers sgRNA-5-BamHI (AGGTGGA TCCTGCTTACCGTAACTTGAAAGTATT) and sgRNA-3-KpnI (AGGTGGTACCAAAAATCTCGCCAACAAGTTGACG). The NosT fragment was amplified from GFP-p1380N-Cas9/sgRNA: cslob1, using NosT-5-KpnI (AGGTGGTACCGAATTTCCCCG ATCGTTCAAACAT) I and NosT-3-AscI (ACCTGGGCCC GGCGCGCCGATCTAGTAACATAGATGA). Through threeway ligation, BamHI-KpnI-digested sgRNA and KpnI-AscI-cut NosT were constructed into BamHI-AscI-treated p1380N-Cas9 to form p1380N-sgRNA.

From p1380N-sgRNA, the CaMV 35S promoter was amplified using primers CaMV35-5-XhoI (5'-ACTCGAGACTAGTACC ATGGTGGACTCCTCTTAA- $\left.{ }^{\prime}\right)$ and sgRNA-cspds-P1 (5'phosphorylated-GCAATGAATTCCCTCTCCAAATGAAATGA ACTTC- $3^{\prime}$ ), and the sgRNA-NosT fragment was amplified using primers sgRNA-cspds-P2 (5'-phosphorylated- ACTAACC ATATGTTTTAGTACTCTGGAAACAGAAT-3') and NosT3-AscI. Through three-way ligation, XhoI-cut CaMV35S and AscI-digested sgRNA-NosT were inserted into XhoI-AscI-cut GFP-p1380N-SaCas9 to build GFP-p1380NSaCas9/sgRNA:cspds (Figure 1A), which is designed to edit the sequence located 15736 bp downstream of ATG in CsPDS (Supplementary Figure 1A). Similarly, GFPp1380N-SaCas9/sgRNA:cs2g12470 was developed, except using primers sgRNA-cs2g12470-P1 (5'-phosphorylatedGCAAAGCTAACCCCTCTCCAAATGAAATGAACTTC- $3^{\prime}$ )

and sgRNA-cs2g12470-P2 (5'-phosphorylated- AGAGGCC TGAGTTTTAGTACTCTGGAAACAGAAT-3') (Figure 1B). GFP-p1380N-SaCas9/sgRNA:cs2g12470 is designed to target the sequence located 259 bp downstream of ATG in Cs $2 g 12470$ (Supplementary Figure 1A).

Using p1380N-sgRNA as template, the CaMV 35S promoter was PCR-amplified using primers CaMV35-5-XhoI and sgRNACs7g03360-P1 (5' -phosphorylated-GAATATTGCCACAGCCCT CTCCAAATGAAATGAACTTC-3'), and the sgRNA1-NosT fragment was PCR-amplified using primers sgRNA-Cs7g03360P2 (5' - phosphorylated-AAACCATTGTTTTAGTACTCTGGAA ACAGAAT- $3^{\prime}$ ) and NosT-3-AscI. XhoI-cut CaMV35S and AscI-digested sgRNA-NosT were inserted into XhoI-AscI-cut GFP-p1380N-SaCas9 to build GFP-p1380N- SaCas9/35SCs7g03360sgRNA1 through three-way ligation. Using Arabidopsis genome as template, the AtU6-1 was amplified using AtU6-1-5-AscI (5'-AGGTGGCGCGCC TCTTACAGCTTAGAAATCTCAAA- $3^{\prime}$ ) and sgRNACs7g03360-P3 (5'-phosphorylated- CCTCTGATACCCCTCT CCAAATGAAATGAACTTC-3'). Using sgRNA-Cs7g03360P4 (5' -phosphorylated- AAGTAGCAAGAGGTTTTAGTACT CTGGAAACAGAAT-3') and NosT-3-P (5'-AGGTACTAGTCC GATCTAGTAACATAGATGACA- $3^{\prime}$ ), the sgRNA2-NosT fragment was amplified from p1380N-sgRNA. Through three-way ligation, AscI-cut AtU6-1 and non-digested sgRNANosT were inserted into AscI-PmeI-treated GFP-p1380N- 
SaCas9/35S-Cs7g03360sgRNA1 to form GFP-p1380N-SaCas9/ 35S-sgRNA1:AtU6-sgRNA2 (Figure 1C).

Using freeze-thaw method, Agrobacterium tumefaciens EHA105 was transformed by binary vectors GFP-p1380NSaCas9/sgRNA:cspds, GFP-p1380N-SaCas9/sgRNA:cs2g12470 and GFP-p1380N-SaCas9/35S-sgRNA1: AtU6-sgRNA2, respectively. The PCR-positive Agrobacterium colonies were singled out for Xcc-facilitated agroinfiltration or citrus transformation.

\section{Xcc-Facilitated Agroinfiltration in Duncan Grapefruit and Agrobacterium-Mediated Carrizo Citrange Transformation}

Duncan grapefruit (Citrus paradisi) was grown in a greenhouse with temperature ranged from 25 to $30^{\circ} \mathrm{C}$. The plants were pruned for flushing before Xanthomonas citri subsp. citri (Xcc)-facilitated agroinfiltration was perform. The recombinant Agrobacterium cells contained binary vector GFP-p1380N-SaCas9/sgRNA:cspds or GFPp1380N-SaCas9/sgRNA:cs2g12470. The detailed protocol for Xcc-facilitated agroinfiltration in citrus leaves was described in previous work (Jia and Wang, 2014b; Jia et al., 2017). Four days after agroinfiltration, genomic DNA was extract from the treated leaves after GFP fluorescence detection.

The epicotyls of Carrizo citrange were employed as explants to establish transgenic plants by Agrobacterium-mediated transformation. The recombinant Agrobacterium cells harbored binary vector GFP-p1380N-SaCas9/35S-sgRNA1:AtU6-sgRNA2. The number of explants used in these experiments was 5460. On those explants, there were 818 shoots. Out of 818 Carrizo shoots, 12 of them exhibited GFP fluorescence in all their tissues, whereas 31 shoots were chimeric. Only the 12 GFP-positive regenerants were subjected to subsequent grafting for PCR verification and SaCas9/sgRNA-mediated indel analysis.

The transgenic Carrizo citrange was verified by PCR amplification, using primers, AtU6-1-5-AscI and NosP-3-P2 (5' TTGTCGTTTCCCGCCTTCAGT-3').

\section{GFP Detection}

GFP-p1380N-SaCas9/35S-sgRNA1:AtU6-sgRNA2-transformed Carrizo citrange was observed under illumination of the Stereo Microscope Fluorescence Adapter (NIGHTSEA) by using a Zeiss Stemi SV11 dissecting microscope, which is equipped with an Omax camera. The transgenic plant leaves were photographed by using the Omax Toupview software.

\section{Cs7g03360 Sequencing and Analysis}

Genomic DNA was extracted from wild type (WT) Carrizo citrange, transgenic Carrizo plants, or the Duncan leaves treated by Xcc-facilitated agroinfiltration. To figure out the conserved region of Cs7g03360 in Carrizo, PCR was performed with the Phusion DNA polymerase (New
England Biolabs) and a pair of primers, Cs7g03360-5-P1 $\left(5^{\prime}\right.$-TATCAGTACCAGTACCAGCAACAT- $\left.3^{\prime}\right)$ and Cs7g033603-P2 (5'-TCCATATTAAGACGAAGATTCCCCAGT-3') (Supplementary Figure 1B). After sequencing, seven potential sgRNAs were found in the conserved sequences, and two were singled out as SaCas9/sgRNA-directed targets, which were adjacent to PAM 5'NNGRRT (Supplementary Figure 1B). The conserved sequences were chosen as targets to target both allele of Cs7g03360 by the same sgRNA. Importantly, the sgRNA-targeting regions locate in the first exon of Cs7g03360 (Supplementary Figure 1).

\section{Selective PCR Amplification of Mutagenized CsPDS and Cs2g12470}

To test the GFP-p1380N-SaCas9/sgRNA:cspds-mediated indels to CsPDS gene, $600 \mathrm{ng}$ of Duncan genomic DNA, extracted from leaves treated by GFP-p1380N-SaCas9/sgRNA:cspds, was digested with NdeI overnight. Using the NdeI-cut genomic DNA as template, PCR was performed using primers CsPDS-5P5 (5'-TGGCAATGTGATTGACGGAGATGC-3') and CsPDS3-P6 (5'-ATGAGTCCTCCTTGTTACTTCAGT-3'), flanking the targeted site of CsPDS.

Similarly, using StuI-digested Duncan genomic DNA prepared from leaves treated by GFP-p1380N-SaCas9/ sgRNA:cs2g12470, the SaCas9/sgRNA-mediated modification was analyzed with a pair of primers Cs2g12470-5-P1 (5'AGCTGATAGGCTTGTGCTTCAG-3') and Cs2g12470-3-P2 (5'-AGGCATCTGGAATGAACCCAGA-3').

The PCR products were ligated to the PCR-BluntII-TOPO vector (Life Technologies), and 16 random colonies were chosen for DNA sequencing. Chromas Lite program was employed to analyze the sequencing results.

\section{Targeted Next Generation Sequencing Analysis}

Genomic DNA from 12 transgenic plants was used as template for PCR amplification using a pair of primers, Cs7g033605-P1 and Cs7g03360-5-P2. All PCR products were pooled to construct the DNA library for sequencing using an Illumina HiSeq 2500 platform at Novogene (Beijing, China). More than 50,000 paired-end reads were generated for each sample. The raw amplicon sequencing reads have been deposited in NCBI Bioproject database under the accession number PRJNA416781. The high quality reads were generated from the raw reads by de-multiplex, barcode and primer deletion using custom Perl script. To further guarantee the quality for each reads, the high quality reads were quality trimmed using sickle software with parameters average quality 30 and reads length threshold 200 bp (Fass et al., 2011). The remaining high-quality reads were clustered with a threshold of $100 \%$ pairwise identity using UCLUST (Edgar, 2010). The representative sequences from abundant clusters with relative abundance $>1 \%$ were aligned using MEGA7 (Tamura et al., 2013) and further be analyzed for indel mutation genotype. 


\section{RESULTS}

\section{Editing CsPDS and Cs2g12470 in Duncan Grapefruit through Transient Expression of SaCas9/sgRNA via Xcc-Facilitated Agroinfiltration}

Though SaCas9/sgRNA has been used to modify Arabidopsis, tobacco and rice genome (Steinert et al., 2015; Kaya et al., 2016), it has not been used in citrus. Previously, Xcc-facilitated agroinfiltration was employed as rapid functional analysis of transgenes in citrus leaves, including SpCas9/sgRNA system (Jia and Wang, 2014b). Here, Xcc-facilitated agroinfiltration was employed to test whether SaCas9/sgRNA could be used to conduct citrus genome editing in Duncan grapefruit. Binary vectors GFP-p1380N-SaCas9/sgRNA:cspds and GFP-p1380NSaCas9/sgRNA:cs2g12470 were constructed to edit CsPDS and Cs2g12470 of Duncan grapefruit, respectively (Figures 1A,B and Supplementary Figure 1A). It should be pointed out that the sgRNA-targeting region of CsPDS harbors NdeI (Figure 1A), and the sgRNA-targeting region of Cs2g12470 contains StuI (Figure 1B). Both NdeI and StuI locate in the predicted SaCas9/sgRNA-cleavage site (Ran et al., 2015), which is necessary for selective PCR amplification. Using either NdeI-digested or StuI-digested genome as template, selective PCR amplification of mutagenized CsPDS and Cs2g12470 was performed to assess SaCas9/sgRNA-mediated mutagenesis after Xcc-facilitated agroinfiltration. The sequencing results of 16 random colonies confirmed that both CsPDS and Cs2g12470 were mutated via SaCas9/sgRNA-mediated mutations (Figure 2), and the mutation occurred 3 bp upstream of the PAM sequence. It should be pointed out that the PAM sequence in CsPDS is $5^{\prime}$ GTGGAT, which is a canonical SaCas9 PAM 5'NNGRRT, whereas in Cs2g12470 it is 5'TAGAGA, which belongs to the non-canonical PAM sequence (Ran et al., 2015; Kaya et al., 2016). Our results are consistent with the previous studies, in which SaCas9/sgRNA was reported to be capable of editing the sequence upstream of both the canonical and non-canonical PAMs (Ran et al., 2015; Kaya et al., 2016).

\section{Modification of Cs7g03360 in Carrizo Citrange via Transgenic Expression of SaCas9/sgRNA}

To generate citrus plants with homozygous mutations, we designed a binary vector to target Cs7g03360, a homologous gene of tomato Argonaute7 (SlAgo7), of Carrizo citrange. The homozygous mutant tomato, created by SpCas9/sgRNAmediated disruption of SlAgo7, has the clear phenotype represented by the first leaves having leaflets without petioles and later-formed leaves lacking laminae (Brooks et al., 2014). Carrizo citrange leaf is composed of three leaflets, which is different from single leaf of Duncan grapefruit. Therefore it is reasonable to assume that easily detectable phenotypic changes will appear in Carrizo leaf, given that homozygous mutant Carrizo could be established by SaCas9/sgRNA-mediated Cs $7 g 03360$ targeting.

Sequence of Cs7g03360 was confirmed by sequencing and sgRNA was designed to target two conserved regions of Cs7g03360 in Carrizo citrange to generate deletion (Supplementary Figure 1B). The binary vector GFP-p1380N-SaCas9/35S-sgRNA1:AtU6-sgRNA2 was constructed to modify the two sites, which were designated as Site 1 and Site 2 (Figure 1C and Supplementary Figure 1B). Through Agrobacterium-mediated epicotyl transformation, 12

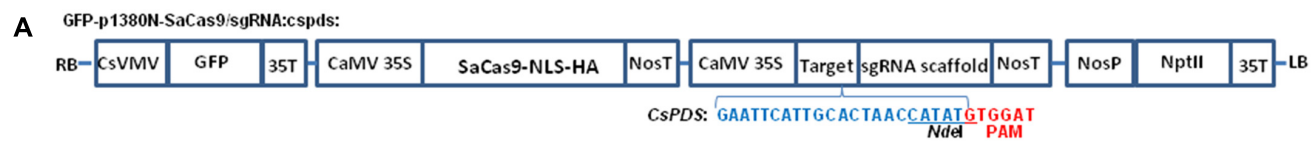

B

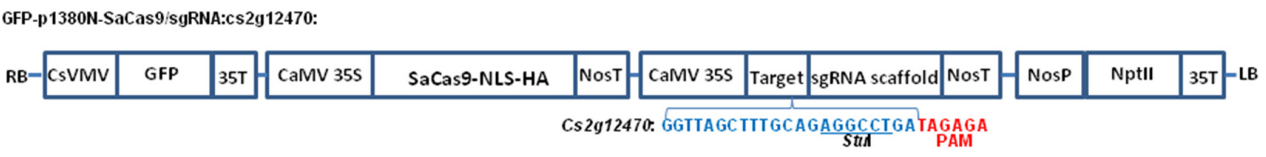

C GFP-p1380N-SaCas9/35S-sgRNA1:AtU6-sgRNA2:

\begin{tabular}{|c|c|c|c|c|c|c|c|c|c|c|c|c|}
\hline $\mathrm{RB}-\mathrm{CsVMV}$ & GFP & $35 T$ & CaMv $35 \mathrm{~s}$ & SaCas9-NLS-HA & NosT & CaMv $35 \mathrm{~s}$ & Targets & sgRNA scaffold & NosT & NosP & NptII & $35 \mathrm{~T}$ \\
\hline & & & \multicolumn{7}{|c|}{ Cs7g03360Site1: GCTGTGGCAATATTCAAACCATTTTGGGT } & & & \\
\hline & & & & & & AtuG-1 & Target & sgRNA scaffold & Nost & & & \\
\hline
\end{tabular}

FIGURE 1 | The schematic diagram of binary vectors. (A) Schematic diagram of p1380N-SaCas9/sgRNA:cspds. A 22 bp sgRNA was used to target CsPDS coding region, which contains Ndel. (B) Schematic diagram of GFP-p1380N-SaCas9/sgRNA:cs2g12470. A 22 bp sgRNA was employed to target Cs2g12470 coding region, which contains Stul. (C) Schematic diagram of GFP-p1380N-SaCas9/35S-sgRNA1:AtU6-sgRNA2. A pair of 23 bp sgRNAs were designed to edit Site 1 and Site 2 of Cs7g03360. CsVMV, the cassava vein mosaic virus promoter; GFP, green fluorescent protein; CaMV 35S and 35T, the cauliflower mosaic virus 35S promoter and its terminator; AtU6, Arabidopsis U6-1 promoter; SaCas9-NLS-HA, the Cas9 endonuclease containing nuclear location signal and HA tag at its C-terminal; targets were highlighted by blue. PAM, protospacer-adjacent motif, highlighted by red; sgRNA scaffold, a synthetic single-guide RNA composed of a fusion of CRISPR RNA and trans-activating CRISPR RNA; NosP and NosT, the nopaline synthase gene promoter and its terminator; Nptll, neomycin phosphotransferase II; LB and RB, the left and right borders of the T-DNA region. 


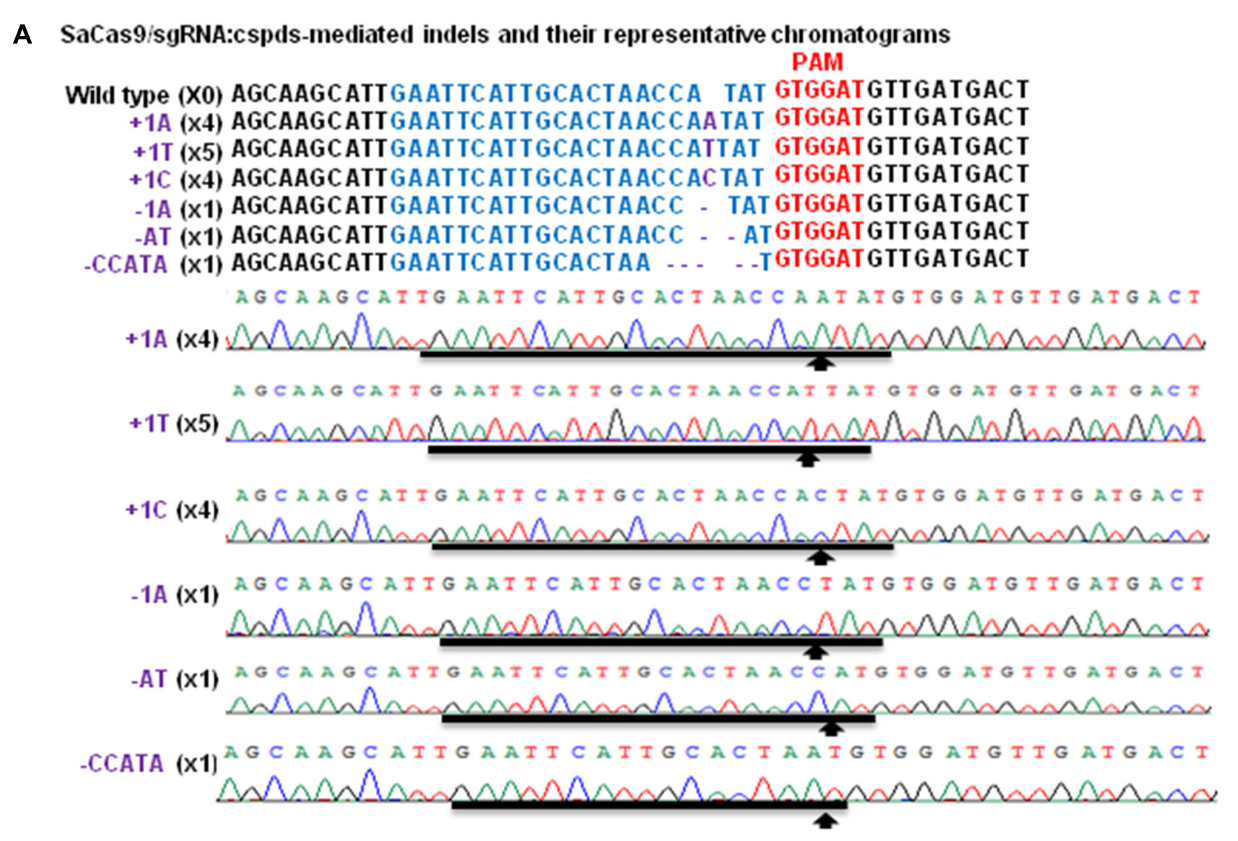

B SaCas9/sgRNA:cs2g12470-mediated indels and their representative chromatograms

PAM

Wild type (X0) AGAGTTACTGGGTTAGCTTTGCAGAGGCC TGA TAGAGAGCTCGgAATG

+1A (x14) AGAGTTACTGGGTT AGCTTTGCAGAG GCCATGA TAGAGAGCTCGGAATG

+1C (x2) AGAGTTACTGGGTTAGCTTTGCAGAGGCCCTGA TAGAGAGCTCGGAATG

$+1 A(x 14)$ AG AGTIACIGGGITAGCIITGCAGAGGCCAIGAIAGAGAGCICGGAAIG

$+1 C(x 2)$ AG AGTIACTGGGTIAGCIITGCAGAGGCCCIGATAGAGAGCTIGGAATG

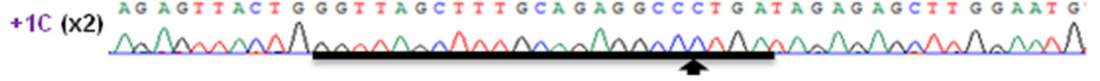

FIGURE 2 | Targeted mutations induced by SaCas9/sgRNA in CsPDS and Cs2g12470. The representative chromatograms of CsPDS and mutations in the CsPDS gene in Duncan grapefruit, after $X_{C c}$-facilitated agroinfiltration. Among 16 colonies sequenced, there are four $+1 A$ and $+1 C(X 4)$, five $+1 T(X 5)$ and one $-1 A,-A T$, -CCATA (X1). (A) The representative chromatograms of Cs2g12470 and mutations in Cs2g12470. Among 16 colonies sequenced, there are 14 +1A (X14) and two +1C (X2). (B) Targeted sequences of CsPDS and Cs2g12470 are highlighted by blue; PAM is in red, and indels are in purple.

GFP-p1380N-SaCas9/35S-sgRNA1:AtU6-sgRNA2-transformed Carrizo plants were created, which were subsequently verified by GFP fluorescence and PCR (Figure 3).

The transgenic Carrizo was subjected to targeted nextgeneration sequencing (NGS) to analyze mutation genotypes and indel rate, mediated by SaCas9/sgRNA. The NGS results indicated that no large fragment deletion between Site 1 and Site 2 took place for all of Carrizo transformants, though SpCas9/sgRNA was successfully used to delete large fragment between two sgRNAs in some plant species (Ma et al., 2015). On the other hand, indel mutations mediated by SaCas9/sgRNA were observed for individual Site 1 and Site 2, as expected, occurring at $3 \mathrm{bp}$ upstream of the PAM site (Figure 4), same as SpCas9/sgRNA-mediated mutations (Jia et al., 2016, 2017). Since the genotype of Site 1 and Site 2 could be either WT or Indel, there were four kinds of genotype combinations in transgenic Carrizo, (1) WT1/...WT2: both Site 1 and Site 2 are WT, (2) WT1/.../Indel2: Site 1 is WT, whereas Site 2 is indel, (3) Indel1/...WT2: Site 1 is indel, whereas Site 2 is WT, (4) Indel1/.../Indel2: both Site 1 and
Site 2 are indel (Figures $\mathbf{4}, \mathbf{5 A}$ ). It should be noted that Site 1 mutation genotypes were either $1 \mathrm{~A}$ insertion or $1 \mathrm{~T}$ insertion, whereas Site 2 mutation genotypes included 1A insertion, $1 \mathrm{~T}$ insertion, and a variety of short deletions (Figures 4, 5A), which indicated that all Carrizo transformants were chimeric.

Based on targeted NGS results, the mutation rates of 12 Carrizo transformants were calculated. Including both Site 1 and Site 2, the rate of genotype WT1/.../WT2 was from $20.11(\mathrm{Cz} 3)$ to $49.60 \%(\mathrm{Cz} 9)$, that of WT1/.../Indel2 was from $13.23(\mathrm{Cz} 7)$ to $53.42 \%(\mathrm{Cz} 3)$, that of Indel1/. . /WT2 was from 0.07 (Cz10) to $2.16 \%(\mathrm{Cz} 2)$, that of Indel1/.../Indel2 was from 14.57 (Cz12) to 38.42\% (Cz7) (Figure 5A). When Cs7g03360 Site 1 was calculated alone, the mutation rates were from $15.55(\mathrm{Cz} 12)$ to $39.13 \%$ (Cz7) (Figure 5B). In the case that only Cs7g03360 Site 2 was calculated, the mutation rates were from $49.01(\mathrm{Cz} 9)$ to $79.67 \%$ (Cz3) (Figure 5C). Therefore, the mutation rate was different for each Carrizo transgenic line. Consistently, the SpCas9/sgRNAtransformed Duncan grapefruit also had different mutation rates (Jia et al., 2016, 2017). 

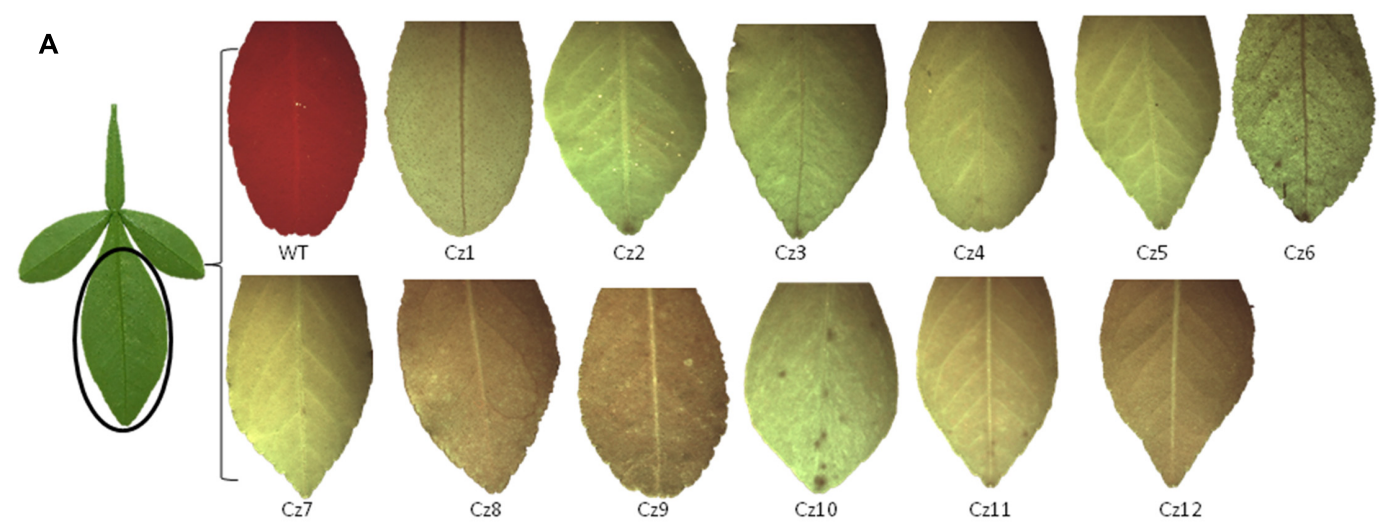

B

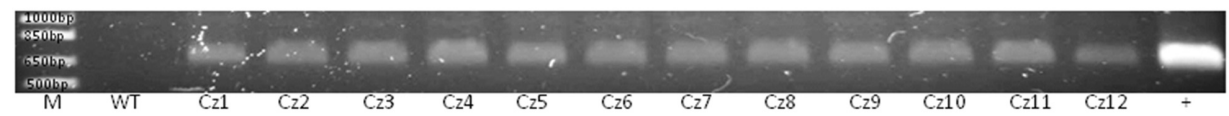

FIGURE 3 | GFP observation and PCR analysis of transgenic Carrizo citrange. (A) Twelve GFP-p1380N-SaCas9/35S-sgRNA1:AtU6-sgRNA2-transformed Carrizo plants (\#Cz1-12) were established through Agrobacterium-mediated epicotyl transformation, which expressed GFP. (B) PCR was performed using primers AtU6-1-5-Ascl and NosP-3-P2. M, 1 kb DNA ladder; WT, wild type; +, GFP-p1380N-SaCas9/35S-sgRNA1:AtU6-sgRNA2 was used as a positive control.

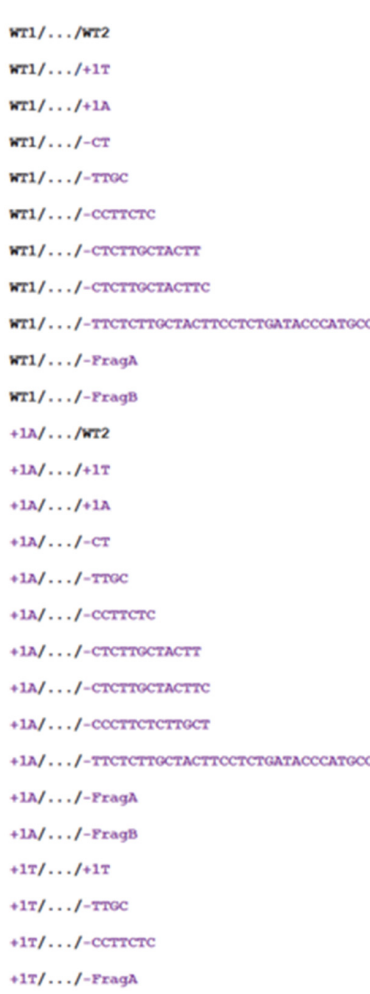

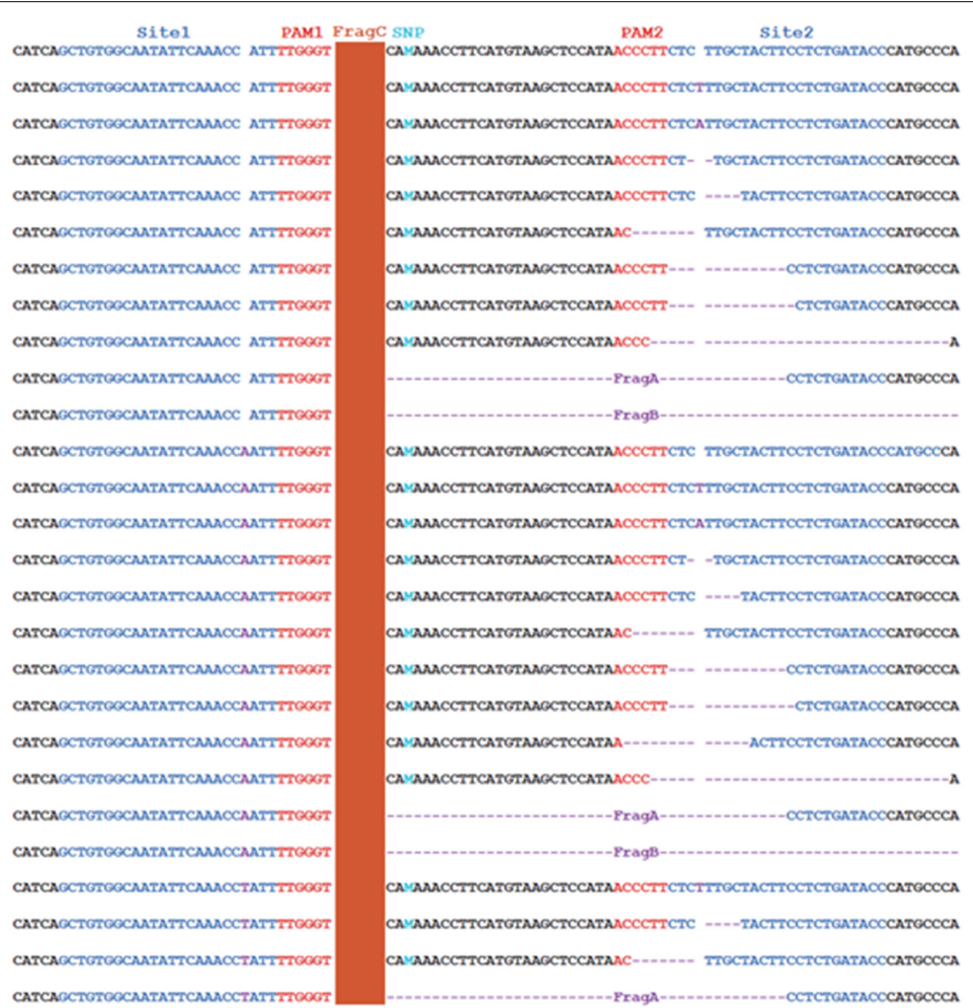

FIGURE 4 | SaCas9/sgRNA-mediated mutation genotypes in 12 Carrizo transformants Representative indel mutation genotypes in Carrizo gene Cs7g03360. The Site 1 mutation only contained $1 \mathrm{bp}$ insertion, and the Site 2 mutations contained $1 \mathrm{bp}$ insertion and short deletions. It should be noted that the FragA deletion and FragB deletion removed the PAM of Site 2. SNP, single nucleotide polymorphism; FragA: CACAAACCTTCATGTAAGCTCCATAACCCTTCT CTTGCTACTT; FragB: TGTAAGCTCCATAACCCTTC. . TTATTATTATITAAATGCA; FragC: TCTGTAACCAAAACCAGCAT. . TTGTATCAAAAACCCATTTG.

Cs7g03360 function is unknown in Citrus. SlAgo, a homologous gene of Cs7g03360 in tomato, affects leaf development. Loss of its function in homozygous tomato leads to leaf abnormality (Brooks et al., 2014). In this study, there were no any visible phenotypes among all transgenic Carrizo plants (Supplementary Figure 2), which might be attributed to none of 


\section{\begin{tabular}{|l|l|l|l|l|l|l|l|l|l|l|l|l|l|l|l}
$\mathbf{A}$ & Cz1 & Cz2 & Cz3 & Cz4 & Cz5 & Cz6 & Cz7 & Cz8 & Cz9 & Cz10 & Cz11 & Cz12
\end{tabular} \begin{tabular}{|l|l|l|l|l|l|l|l|l|l|l|l|l|l|}
\hline WT1/.../WT2 & $33.48 \%$ & $26.31 \%$ & $20.11 \%$ & $29.93 \%$ & $34.28 \%$ & $21.54 \%$ & $47.64 \%$ & $45.34 \%$ & $49.60 \%$ & $29.23 \%$ & $40.39 \%$ & $44.92 \%$ \\
\hline
\end{tabular}

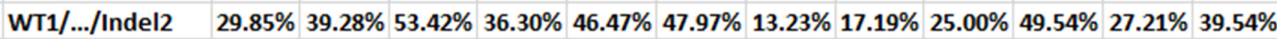 \begin{tabular}{|l|l|l|l|l|l|l|l|l|l|l|l|l|l|}
\hline Indel1/.../WT2 & $1.07 \%$ & $2.16 \%$ & $0.22 \%$ & $0.68 \%$ & $1.17 \%$ & $0.28 \%$ & $0.72 \%$ & $0.94 \%$ & $1.39 \%$ & $0.07 \%$ & $0.41 \%$ & $0.98 \%$ \\
\hline
\end{tabular}

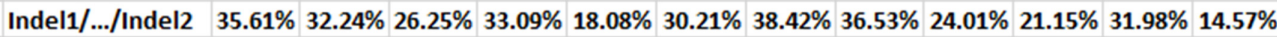

B \begin{tabular}{|l|l|l|l|l|l|l|l|l|l|l|l}
\hline Cz1 & Cz2 & Cz3 & Cz4 & Cz5 & Cz6 & Cz7 & Cz8 & Cz9 & Cz10 & Cz11 & Cz12 \\
\hline
\end{tabular}

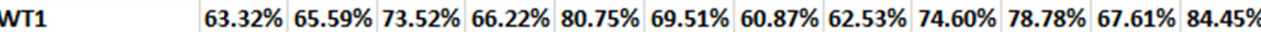

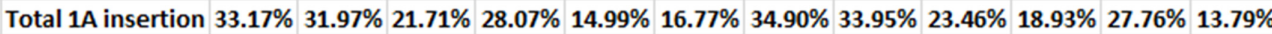
\begin{tabular}{|l|l|l|l|l|l|l|l|l|l|l|l|l|}
\hline Total 1T insertion & $3.51 \%$ & $2.44 \%$ & $4.77 \%$ & $5.71 \%$ & $4.26 \%$ & $13.72 \%$ & $4.23 \%$ & $3.52 \%$ & $1.94 \%$ & $2.29 \%$ & $4.63 \%$ & $1.76 \%$ \\
\hline
\end{tabular}

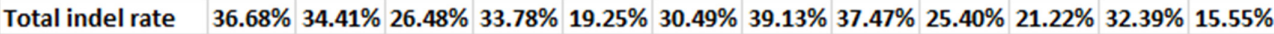

\begin{tabular}{|c|c|c|c|c|c|c|c|c|c|c|c|c|}
\hline & Cz1 & $\mathrm{Cz} 2$ & $\mathrm{Cz3}$ & Cz4 & Cz5 & Cz6 & $\mathrm{Cz} 7$ & $\mathrm{Cz8}$ & Cz9 & Cz10 & Cz11 & Cz12 \\
\hline WT2 & $34.54 \%$ & $28.47 \%$ & $20.33 \%$ & $30.61 \%$ & $35.45 \%$ & $21.82 \%$ & $48.36 \%$ & $46.27 \%$ & $50.99 \%$ & $29.30 \%$ & $40.81 \%$ & $45.90 \%$ \\
\hline Total & $12 \%$ & $.05 \%$ & $2.66 \%$ & $1 \%$ & $2.84 \%$ & $1.72 \%$ & $3.63 \%$ & $6.34 \%$ & $3.56 \%$ & $1.67 \%$ & $2.60 \%$ & \\
\hline Tot: & $\%$ & 32.5 & $60.81 \%$ & $63.13 \%$ & $53.22 \%$ & $75.62 \%$ & 26. & 37. & $\%$ & $\%$ & $\%$ & $\%$ \\
\hline & & $34.97 \%$ & $16.20 \%$ & $75 \%$ & $8.50 \%$ & $0.84 \%$ & $21.70 \%$ & $9.54 \%$ & $14.91 \%$ & $31.78 \%$ & $11.30 \%$ & $17.22 \%$ \\
\hline Total indel rate & $65.46 \%$ & $71.53 \%$ & $79.67 \%$ & $69.39 \%$ & $64.55 \%$ & $78.18 \%$ & $51.64 \%$ & $53.73 \%$ & $49.01 \%$ & $70.70 \%$ & $59.19 \%$ & $54.10 \%$ \\
\hline
\end{tabular}

FIGURE 5 | Indel mutation rates in 12 Carrizo transformants. (A) Mutation rates of both Site 1 and Site 2 for 12 transgenic Carrizo lines. Twelve Carrizo transformants had different mutation rate, based on next generation sequencing results. The mutation rates were from 14.57 (Cz12) to 38.42\% (Cz7). (B) Mutation rates of Cs7g03360 Site 1 for 12 transgenic Carrizo lines. The mutation rates were from 15.55 (Cz12) to 39.13\% (Cz7). (C) Mutation rates of Cs7g03360 Site 2 for 12 transgenic Carrizo lines. The mutation rates were from 49.01 (Cz9) to 79.67\% (Cz3).

transgenic Carrizo containing Cs7g03360 homozygous mutation (Figures 4, 5). In SaCas9/sgRNA-transformed Arabidopsis, the indel mutations were heritable, and the homozygous plants were created in next generation (Steinert et al., 2015). It is possible to obtain the homozygous Cs7g03360-mutated Carrizo in the $\mathrm{T}_{1}$ generation, which is useful to study Cs7g03360 function in citrus. Alternatively, the transgenic Carrizo could be harnessed as either maternal or paternal donor to produce Cs7g03360-knock-out plants in next generation through hybridizing with other citrus cultivar. However, to create homozygous Carrizo transformants in the $\mathrm{T}_{0}$ generation via CRISPR/Cas9 still remains a great challenge for citrus genome editing. In a recent study, YAO promoter was harnessed to drive SpCas9 expression to promote indel efficiency in citrus (Zhang et al., 2017). It is reported that the Csy4 ribonuclease and tRNA processing enzymes can be used to simultaneously express 12 sgRNAs for efficient indel mutations, and the TREX2 exonuclease can also enhance mutagenesis (Cermak et al., 2017).

Using a web tool ${ }^{1}$ (Bae et al., 2014), potential off-target mutagenesis induced by SaCas9/35S-sgRNA1:AtU6-sgRNA2 was filtered (Mismatch number $=3$, RNA bulge size $=1$ ), based on sweet orange genome. There was no off-targets for Cs7g03360 Site 1, on the other hand, three potential off-targets of Cs7g03360 Site 2 were identified (Supplementary Figure 3 ). The three potential off-targets were subjected to PCR-amplification, and sub-cloning. Five colonies for each off-target were randomly chosen for sequencing. The results demonstrated that no mutations took place in the three potential off-targets in the transgenic Carrizo plants (Supplementary Table 1).

\section{DISCUSSION}

Our results clearly show that SaCas9/sgRNA can be used to modify citrus genome via either transient expression or transgenic expression. It was reported that the genome editing of Arabidopsis thaliana mediated by SaCas9/sgRNA is comparable to SpCas9/sgRNA (Steinert et al., 2015). We have previously used SpCas9/sgRNA to modify citrus genome (Jia and Wang, 2014a; Jia et al., 2016, 2017). It appears that the genome editing of citrus mediated by SaCas9/sgRNA is comparable to SpCas9/sgRNA. Importantly, SaCas9 is 1053 aa nuclease, much smaller than SpCas9 (1368 aa), which renders SaCas9 easier to handle and transform of target cells. To bind to its target sequence, the Cas9 nuclease also requires the PAM sequence. SaCas9 recognizes NNGRRT whereas SpCas9 recognizes NGG (Anders et al., 2014; Steinert et al., 2015). Thus, successful application of SaCas9 in citrus genome editing has expanded the target repertoire of Cas9 in citrus. It should be feasible to simultaneously employ SaCas9 and SpCas9 recognizing different sequence motifs in the same citrus cell. Furthermore, different SaCas9 orthologues could be developed to switch on one gene and off another in citrus, since $\mathrm{dSpCas} 9$ has been used to activate or repress gene transcription in plants (Piatek et al., 2015). The PAM sequence of SaCas9 is predicted to occur once every $32 \mathrm{bp}$, compared to once every 8 bp for SpCas9 (Kleinstiver et al., 2015). According to fuzznuc commandline analysis from EMBOSS package ${ }^{2}$, in sweet orange the PAM sequence of SaCas9 occurs once every 79 bp and every 32 bp for SpCas9. Thus, SaCas9/sgRNA system has potential to improve the specificity of genome editing and reduce off-target.

${ }^{1}$ http://www.rgenome.net/cas-offinder/

${ }^{2}$ http://emboss.sourceforge.net/ 
No off-targets were observed for citrus genome editing in our study. In transgenic tobacco, SaCas9/sgRNA-mediated off-targets have not been detected either (Kaya et al., 2016).

Most of mutation genotypes of SaCas9/sgRNA in citrus was $1 \mathrm{bp}(1 \mathrm{~A}$ or $1 \mathrm{~T}$ ) insertion (Figures 4, 5), which are similar to those of SpCas9/sgRNA-mediated modification (Jia et al., 2016, 2017). It was previously reported that there were long deletions of up to $50 \mathrm{bp}$ in SaCas9/sgRNAtransformed Arabidopsis and long deletions up to $57 \mathrm{bp}$ in SaCas9/sgRNA-transformed rice (Steinert et al., 2015; Kaya et al., 2016). In this study, the long deletion of 141 bp was observed (Figure 4), which is longer than the deletion of 127 bp in SpCas9/sgRNA-transformed rice (Kaya et al., 2016). The mechanism underlying the long deletion is unknown. Intriguingly, the Indel 2 rate was much higher than that of Indel1 (Figures 5B,C), and the mutation genotypes of Indel2 was more variable than those of Indell (Figure 4). We reason that the differences might result from that CaMV $35 \mathrm{~S}$ was used to drive Cs7g03360sgRNA1, AtU6-1 for Cs7g03360sgRNA2. In transgenic citrus, both $35 \mathrm{~S}$ and $\mathrm{U} 6$ have been successfully employed to promote the transcription of sgRNA (Jia et al., 2017; Peng et al., 2017; Zhang et al., 2017). Though the mutation efficiency is up to $100 \%$ in two study employing U6 (Peng et al., 2017; Zhang et al., 2017), further work is necessary to answer which promoter is more efficient. On the other hand, Cs7g03360sgRNA1 recognizes the positive strand of DNA, Cs7g03360sgRNA2 recognizes the negative strand, we could not rule out the effect of positive strand and negative strand on the efficacy of genome editing. It has been suggested that CRISPR/Cas9 is more efficient when sgRNA is targeting the negative strand than targeting the positive strand (Bortesi et al., 2016). In addition, the GC content is different between

\section{REFERENCES}

Altenbuchner, J. (2016). Editing of the Bacillus subtilis genome by the CRISPR-Cas9 system. Appl. Environ. Microbiol. 82, 5421-5427. doi: 10.1128/AEM.01453-16

Anders, C., Niewoehner, O., Duerst, A., and Jinek, M. (2014). Structural basis of PAM-dependent target DNA recognition by the Cas 9 endonuclease. Nature 513, 569-573. doi: 10.1038/nature13579

Bae, S., Park, J., and Kim, J. S. (2014). Cas-OFFinder: a fast and versatile algorithm that searches for potential off-target sites of Cas9 RNA-guided endonucleases. Bioinformatics 30, 1473-1475. doi: 10.1093/bioinformatics/btu048

Bortesi, L., Zhu, C., Zischewski, J., Perez, L., Bassié, L., Nadi, R., et al. (2016). Patterns of CRISPR/Cas9 activity in plants, animals and microbes. Plant Biotechnol. J. 14, 2203-2216. doi: 10.1111/pbi.12634

Brooks, C., Nekrasov, V., Lippman, Z. B., and Van Eck, J. (2014). Efficient gene editing in tomato in the first generation using the clustered regularly interspaced short palindromic repeats/CRISPR-associated 9 system. Plant Physiol. 166, 1292-1297. doi: 10.1104/pp.114.247577

Cermak, T., Curtin, S. J., Gil-Humanes, J., Cegan, R., Kono, T. J. Y., Konecna, E., et al. (2017). A multipurpose toolkit to enable advanced genome engineering in plants. Plant Cell 29, 1196-1217.

Cho, S. W., Kim, S., Kim, J. M., and Kim, J. S. (2013). Targeted genome engineering in human cells with the Cas9 RNA-guided endonuclease. Nat. Biotechnol. 31, 230-232. doi: 10.1038/nbt.2507

Davey, M. R., Anthony, P., Power, J. B., and Lowe, K. C. (2005). Plant protoplasts: status and biotechnological perspectives. Biotechnol. Adv. 23, 131-171. doi: 10.1016/j.biotechadv.2004.09.008
Cs7g03360sgRNA1 and Cs7g03360sgRNA2, which has a notable effect on CRISPR/Cas9-mediated mutation efficiency (Bortesi et al., 2016).

\section{CONCLUSION}

We have clearly shown that SaCas9 can be used to modify the citrus genome. Further optimization of SaCas9/sgRNA system is needed to increase the mutation rate, including SaCas9 expression driven by Yao promoter, multiple sgRNAs expressed via the Csy4 ribonuclease and tRNA processing enzymes.

\section{AUTHOR CONTRIBUTIONS}

HJ and NW wrote the manuscript. HJ, JX, YZ, and VO performed the experiments. All authors read and approved the manuscript.

\section{ACKNOWLEDGMENT}

This study has been supported by Florida Citrus Initiative Program and USDA NIFA AFRI Plant Biotic Interactions Program (2017-02986).

\section{SUPPLEMENTARY MATERIAL}

The Supplementary Material for this article can be found online at: https://www.frontiersin.org/articles/10.3389/fpls.2017.02135/ full\#supplementary-material

Edgar, R. C. (2010). Search and clustering orders of magnitude faster than BLAST. Bioinformatics 26, 2460-2461. doi: 10.1093/bioinformatics/btq461

Fass, J. N., Joshi, N. A., Couvillion, M. T., Bowen, J., Gorovsky, M. A., Hamilton, E. P., et al. (2011). Genome-scale analysis of programmed DNA elimination sites in Tetrahymena thermophila. G3 1, 515-522. doi: 10.1534/g3.111.00 0927

Garneau, J. E., Dupuis, M., Villion, M., Romero, D. A., Barrangou, R., Boyaval, P., et al. (2010). The CRISPR/Cas bacterial immune system cleaves bacteriophage and plasmid DNA. Nature 468, 67-71. doi: 10.1038/nature09523

Gottwald, T. R. (2010). Current epidemiological understanding of citrus huanglongbing. Annu. Rev. Phytopathol. 48, 119-139. doi: 10.1146/annurevphyto-073009- 114418

Horvath, P., and Barrangou, R. (2010). CRISPR/Cas, the immune system of bacteria and archaea. Science 327, 167-170. doi: 10.1126/science.1179555

Jia, H., Orbovic, V., Jones, J. B., and Wang, N. (2016). Modification of the PthA4 effector binding elements in Type I CsLOB1 promoter using Cas9/sgRNA to produce transgenic Duncan grapefruit alleviating Xcc $\Delta$ pthA4:dCsLOB1.3 infection. Plant Biotechnol. J. 14, 1291-1301. doi: 10.1111/pbi.12495

Jia, H., and Wang, N. (2014a). Targeted genome editing of sweet orange using Cas9/sgRNA. PLOS ONE 9:e93806. doi: 10.1371/journal.pone.0093806

Jia, H., and Wang, N. (2014b). Xcc-facilitated agroinfiltration of citrus leaves: a tool for rapid functional analysis of transgenes in citrus leaves. Plant Cell Rep. 33, 1993-2001. doi: 10.1007/s00299-014-1673-9

Jia, H., Zhang, Y., Orbovic, V., Xu, J., White, F. F., Jones, J. B., et al. (2017). Genome editing of the disease susceptibility gene CsLOB1 in citrus confers resistance to citrus canker. Plant Biotechnol. J. 15, 817-823. doi: 10.1111/pbi.12677 
Jinek, M., Chylinski, K., Fonfara, I., Hauer, M., Doudna, J. A., Charpentier, E., et al. (2012). A programmable dual-RNA-guided DNA endonuclease in adaptive bacterial immunity. Science 337, 816-821. doi: 10.1126/science.1225829

Kaya, H., Mikami, M., Endo, A., Endo, M., and Toki, S. (2016). Highly specific targeted mutagenesis in plants using Staphylococcus aureus Cas9. Sci. Rep. 6:26871. doi: 10.1038/srep26871

Kleinstiver, B. P., Prew, M. S., Tsai, S. Q., Nguyen, N. T., Topkar, V. V., Zheng, Z., et al. (2015). Broadening the targeting range of Staphylococcus aureus CRISPR-Cas9 by modifying PAM recognition. Nat. Biotechnol. 33, 1293-1298. doi: $10.1038 /$ nbt.3404

Li, J. F., Zhang, D., and Sheen, J. (2014). Cas9-based genome editing in Arabidopsis and tobacco. Methods Enzymol. 546, 459-472. doi: 10.1016/B978-0-12-801185$0.00022-2$

Liu, R., Chen, L., Jiang, Y., Zhou, Z., and Zou, G. (2015). Efficient genome editing in filamentous fungus Trichoderma reesei using the CRISPR/Cas9 system. Cell Discov. 1:15007. doi: 10.1038/celldisc.2015.7

Ma, X., Zhang, Q., Zhu, Q., Liu, W., Chen, Y., Qiu, R., et al. (2015). A robustCRISPR/Cas9 system for convenient, high-efficiency multiplex genome editing in monocot and dicot plants. Mol. Plant 8, 1274-1284. doi: 10.1016/j.molp.2015.04.007

Makarova, K. S., Zhang, F., and Koonin, E. V. (2017a). SnapShot: class 1 CRISPRCas systems. Cell 168, 946-946.e1. doi: 10.1016/j.cell.2017.02.018

Makarova, K. S., Zhang, F., and Koonin, E. V. (2017b). SnapShot: class 2 CRISPRCas systems. Cell 168, 328-328.e1. doi: 10.1016/j.cell.2016.12.038

Nishimasu, H., Cong, L., Yan, W. X., Ran, F. A., Zetsche, B., Li, Y., et al. (2015). Crystal structure of Staphylococcus aureus Cas9. Cell 162, 1113-1126. doi: 10.1016/j.cell.2015.08.007

Peng, A., Chen, S., Lei, T., Xu, L., He, Y., Wu, L., et al. (2017). Engineering cankerresistant plants through CRISPR/Cas9-targeted editing of the susceptibility gene CsLOB1 promoter in citrus. Plant Biotechnol. J. 15, 1509-1519. doi: $10.1111 /$ pbi.12733
Piatek, A., Ali, Z., Baazim, H., Li, L., Abulfaraj, A., Al-Shareef, S., et al. (2015). RNA-guided transcriptional regulation in planta via synthetic dCas9-based transcription factors. Plant Biotechnol. J. 13, 578-589. doi: 10.1111/pbi.12284

Ran, F. A., Cong, L., Yan, W. X., Scott, D. A., Gootenberg, J. S., Kriz, A. J., et al. (2015). In vivo genome editing using Staphylococcus aureus Cas9. Nature 520, 186-191. doi: 10.1038/nature14299

Steinert, J., Schiml, S., Fauser, F., and Puchta, H. (2015). Highly efficient heritable plant genome engineering using Cas9 orthologues from Streptococcus thermophilus and Staphylococcus aureus. Plant J. 84, 1295-1305. doi: 10.1111/ tpj. 13078

Tamura, K., Stecher, G., Peterson, D., Filipski, A., and Kumar, S. (2013). MEGA6: molecular evolutionary genetics analysis version 6.0. Mol. Biol. Evol. 30, 2725-2729. doi: 10.1093/molbev/mst197

Wang, N., Pierson, E. A., Setubal, J. C., Xu, J., Levy, J. G., Zhang Y., et al. (2017). The Candidatus Liberibacter-Host interface: insights into pathogenesis mechanisms and disease control. Annu. Rev. Phytopathol. 55, 451-482. doi: 10.1146/annurev-phyto-080516-035513

Zhang, F., LeBlanc, C., Irish, V. F, and Jacob, Y. (2017). Rapid and efficient CRISPR/Cas9 gene editing in Citrus using the YAO promoter. Plant Cell Rep. 36, 1883-1887. doi: 10.1007/s00299-017-2202-4

Conflict of Interest Statement: The authors declare that the research was conducted in the absence of any commercial or financial relationships that could be construed as a potential conflict of interest.

Copyright (c) 2017 Jia, Xu, Orbović, Zhang and Wang. This is an open-access article distributed under the terms of the Creative Commons Attribution License (CC BY). The use, distribution or reproduction in other forums is permitted, provided the original author(s) or licensor are credited and that the original publication in this journal is cited, in accordance with accepted academic practice. No use, distribution or reproduction is permitted which does not comply with these terms. 